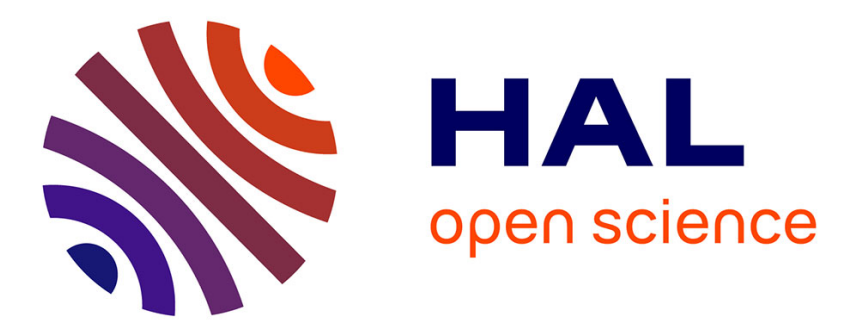

\title{
Viscoelastic shear modulus measurement of thin materials by interferometry at ultrasonic frequencies
}

Alverède Simon, Gautier Lefebvre, Tony Valier-Brasier, Régis Wunenburger

\section{To cite this version:}

Alverède Simon, Gautier Lefebvre, Tony Valier-Brasier, Régis Wunenburger. Viscoelastic shear modulus measurement of thin materials by interferometry at ultrasonic frequencies. Journal of the Acoustical Society of America, 2019, 146 (5), pp.3131 - 3140. 10.1121/1.5131026 . hal-03292337

\section{HAL Id: hal-03292337 \\ https://hal.science/hal-03292337}

Submitted on 20 Jul 2021

HAL is a multi-disciplinary open access archive for the deposit and dissemination of scientific research documents, whether they are published or not. The documents may come from teaching and research institutions in France or abroad, or from public or private research centers.
L'archive ouverte pluridisciplinaire HAL, est destinée au dépôt et à la diffusion de documents scientifiques de niveau recherche, publiés ou non, émanant des établissements d'enseignement et de recherche français ou étrangers, des laboratoires publics ou privés. 
Viscoelastic shear modulus measurement of thin materials by interferometry at ultrasonic frequencies

Alverède Simon, Gautier Lefebvre, Tony Valier-Brasier, and Régis Wunenburger

Citation: The Journal of the Acoustical Society of America 146, 3131 (2019); doi: 10.1121/1.5131026

View online: https://doi.org/10.1121/1.5131026

View Table of Contents: https://asa.scitation.org/toc/jas/146/5

Published by the Acoustical Society of America

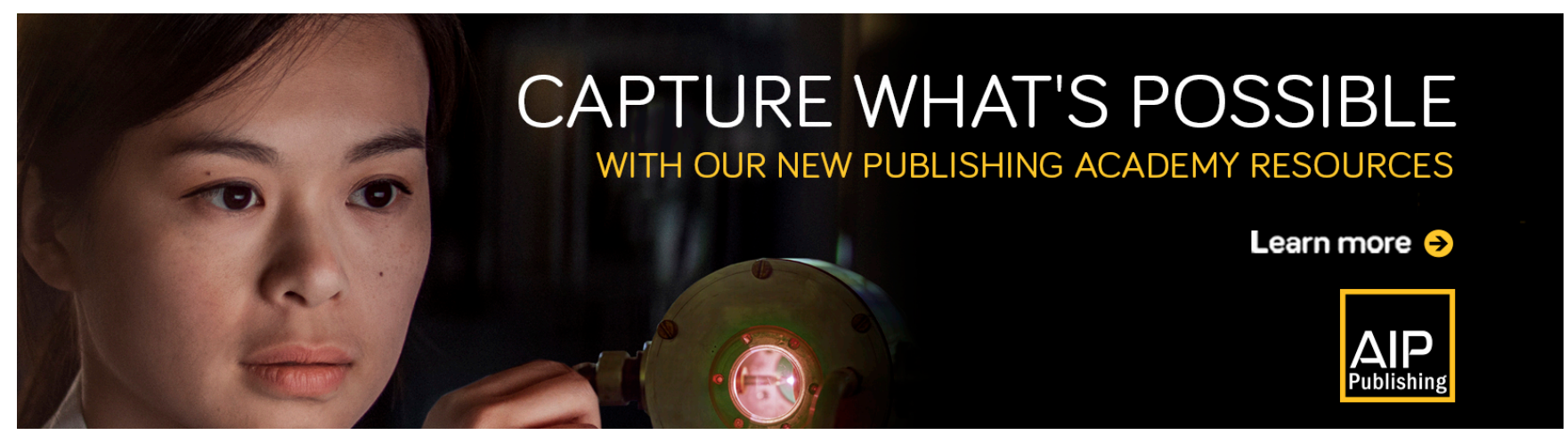




\title{
Viscoelastic shear modulus measurement of thin materials by interferometry at ultrasonic frequencies
}

\author{
Alverède Simon, Gautier Lefebvre, ${ }^{\text {a) }}$ Tony Valier-Brasier, and Régis Wunenburger ${ }^{\text {b) }}$ \\ Sorbonne Université, CNRS, Institut Jean Le Rond d'Alembert, F-75005 Paris, France
}

(Received 12 July 2019; revised 3 October 2019; accepted 8 October 2019; published online 6 November 2019)

This paper presents a technique for measuring the complex shear modulus of thin slabs of viscoelastic solids based on the measurement of the reflection and transmission of plane shear waves through a sample inserted between two delays lines. Reproducible shear wave transmission through the sample is achieved by inserting bond layers with controlled thickness between the delay lines and the sample and by characterizing beforehand the bond rheology. The frequency dependent complex shear modulus is quantitatively evaluated from the transmission and reflection coefficients using an exact model of interferences within the delay line-bond-sample-bond-delay line sandwich and by selecting the solution among the muliple solutions of the inverse problem from considerations on time of flight and sample thickness. Thanks to its reproducibility and accuracy, this method appears as an original and efficient technique for quantitatively characterizing the high frequency shear modulus of attenuating materials. (C) 2019 Acoustical Society of America.

https://doi.org/10.1121/1.5131026

[MRH]

Pages: $3131-3140$

\section{INTRODUCTION}

The complex shear modulus of solid materials at ultrasonic frequencies can be determined by measuring the velocity and attenuation of shear waves through a sample. Materials of interest include viscoelastic materials but also composite materials and metamaterials, whose low-frequency behavior can be described in terms of effective modulus.

Several techniques have been developed to study the propagation of shear waves inside a solid sample: (i) an immersion technique based on the conversion to shear wave of a longitudinal wave incident in an immersed slab beyond critical angle, which has been applied to viscoelastic materials (Hartmann and Jarzynski, 1974; Wu, 1996; Reddy et al., 2005), (ii) a technique based on the generation and propagation of torsional waves along a bar (Simonetti and Cawley, 2004), (iii) contact techniques based on shear-wave transducers (Treiber et al., 2009; Asay et al., 1969) and possibly delay lines (Papadakis, 1968). The main advantage of technique (i) is that liquid wetting ensures a reproducible transmission at the liquid-sample interface. Techniques (ii) and (iii) involve the transmission of shear waves through solidsolid interfaces. Shear wave transmission is obtained by compressing the solid/solid interface (Simonetti and Cawley, 2004) and often by inserting a viscoelastic bond. Achieving an efficient, measurable and ideally reproducible bonding between the sample and the delay line-transducer constitutes a major issue regarding the determination of shear wave attenuation and is thus a bottleneck for metrology.

Relevant information on the velocity and attenuation of shear waves can be retrieved by analyzing either (i) the

\footnotetext{
a) Also at: Université de Technologie de Compiègne, Sorbonne Universités, Laboratoire Roberval de Mécanique, F-60200 Compiègne, France.

b)Electronic mail: regis.wunenburger@sorbonne-universite.fr
}

echoes of wavetrains reflected and transmitted by the sample (Asay et al., 1969; Treiber et al., 2009) or (ii) their interferences (Papadakis, 1968; Simonetti and Cawley, 2004). Method (i) requires that echoes ringing inside the sample shall not overlap. This imposes a lower limit to the central frequency of wavetrains or a minimal sample thickness, which can be problematic in the case of highly absorbing samples. Method (ii) is more versatile but requires a precise model of the multiple reflections inside the layered material composed of delay lines, bond layers and sample. To get around the lack of knowledge of the rheological properties of the bond and of control of the bond layer thickness, the effect of the bond layer on shear wave propagation was formerly measured in situ and injected into the propagation model (Asay et al., 1969; Treiber et al., 2009).

In order to be able to accurately characterize absorbing samples at low frequencies, we developed a different strategy based on (i) the fulfillment of a reproducible and wellcharacterized bonding and (ii) the implementation of an exact model of interferences of a low-frequency wavetrain inside the delay line-bond-sample-bond-delay line assembly, hereafter called the five-layers sandwich. In this article, we present our procedure for obtaining a reproducible and wellcontrolled bonding, the reflection-transmission technique, the interference model and the signal analysis procedure. We demonstrate the accuracy of the resulting technique by characterizing PMMA and comparing our measurements with published data.

\section{EXPERIMENT}

In this section, we present in detail the setup, the experimental protocol, and how the reflection and transmission coefficients are experimentally determined. 


\section{A. Experimental setup}

The principle of the experiment is to measure the shear waves reflected and transmitted by a slab of solid material. Two delay lines are used to separate the different echoes. Literature confirmed by preliminary attempts have led us to the conclusion that controlling the bond layer thickness through tightening is illusory because the final bond layer thickness depends on unreproducible steps that are bond drop-off and tightening history. On the contrary, a welldefined and reproducible contact is obtained by inserting between each delay line and the slab a spacer to control the thickness of the bond layer. A sketch of the experiment is shown in Fig. 1(a).

We use two identical $L=(59.88 \pm 0.03) \mathrm{mm}$ thick parallelepipedic delay lines made of 2017A Dural alloy with mirror-polished faces. A pair of identical shear wave transducers is mounted in contact on each delay line using a small, controlled amount $\left(55 \mu \mathrm{g} \cdot \mathrm{mm}^{-2}\right)$ of Olympus SWC-2 viscoelastic bond. As sketched in Fig. 1(a), a tightening screw applies a constant force on the transducer in order to obtain an efficient transmission of shear waves. The torque applied on these screws is reproducibly set at $(2.0 \pm 0.2) \mathrm{N} \cdot \mathrm{m}$. The Olympus shear wave transducers used in this study are V152-type with $2 a=0.5$ inch active diameter, $2.25 \mathrm{MHz}$ nominal central frequency $\left(f_{\mathrm{c}}=2.22 \mathrm{MHz}\right.$ actual central frequency) and V155-type with $2 a=0.5$ inch active diameter, $5.0 \mathrm{MHz}$ nominal central frequency $\left(f_{\mathrm{c}}=4.18 \mathrm{MHz}\right.$ actual central frequency).

The solid samples used in this study are made of pure poly(methyl methacrylate) (PMMA) provided by GammaTec (F-Staffelfelden). Two samples have been studied: one $H=(4.888 \pm 0.005) \mathrm{mm}$ thick sample labelled " $5 \mathrm{~mm}$ thick sample," and one $H=(2.974 \pm 0.002)$ mm thick sample labelled "3 mm thick sample" (thicknesses have been measured at $(5.0 \pm 0.2)^{\circ} \mathrm{C}$, see below).

The mechanical contact between the delay lines and the solid sample is achieved by inserting between each delay line and the slab a thin washer that plays the role of a spacer and filling it with bond. The washer made of brass and $h=(105.0 \pm 1.4) \mu \mathrm{m}$ thick and has $D_{\mathrm{w}}=50 \mathrm{~mm}$ internal diameter. The delay lines, spacers and sample are tightened together using three threaded rods, as sketched in Fig. 1(a).

The setup is put in a refrigerated incubator at $(5.0 \pm 0.2)^{\circ} \mathrm{C}$, which importantly ensures a steady transmission by the bond layer squeezed between the transducer and the delay line after a few hours (at room temperature, this transmission drifts over days). Spike-like pulses are applied to the emitter using an Olympus PR5072-type pulser-receiver at a $100 \mathrm{~Hz}$ rate. Care has been taken to balance the internal resistances of the signal acquisition channels. Voltage signals are acquired and digitized with a 14-bit resolution and at $f_{s}=400 \mathrm{MHz}$ sampling frequency. The acquisitions are averaged over 1000 iterations.

\section{B. Signal acquisition protocol}

Absolute determination of the stress reflection and transmission coefficients $\mathcal{R}$ and $\mathcal{T}$ of the five-layers sandwich can be performed by combining the measurements of the reflections by the Dural-air interface of each isolated delay line [configuration sketched in Fig. 1(b)] and the measurement of the reflection and transmission by the five-layers sandwich [configuration sketched in Fig. 1(a)] according to the following protocol. The setup being mounted as sketched in Fig. 1(a) and the transducers being labelled respectively by 1 and 2 , the signals transmitted by the five-layers sandwich from 1 to $2, S_{t 1}$, and from 2 to $1, S_{t 2}$ are acquired. Then, the signals reflected by the five-layers sandwich from 1 back to $1, S_{r 1}$, and from 2 back to $2, S_{r 2}$, are acquired. Finally, the five-layers sandwich is unmounted as sketched in Fig. 1(b). Then, the calibration signals reflected by the end of each bare delay line from 1 toward $1, S_{c 1}$, and from 2 toward $2, S_{c 2}$, are acquired.

In Fig. 2, which concerns the $5 \mathrm{~mm}$ thick sample and a pulse excitation at $f_{\mathrm{c}}=2.22 \mathrm{MHz}$ central frequency, three signals are shown: a reflection signal, a transmission signal and a calibration signal. In the following, we focus on the reflection signal that has traveled a single round trip across the delay line and on the transmission signal that has traveled a one-way trip across the whole five-layers sandwich.
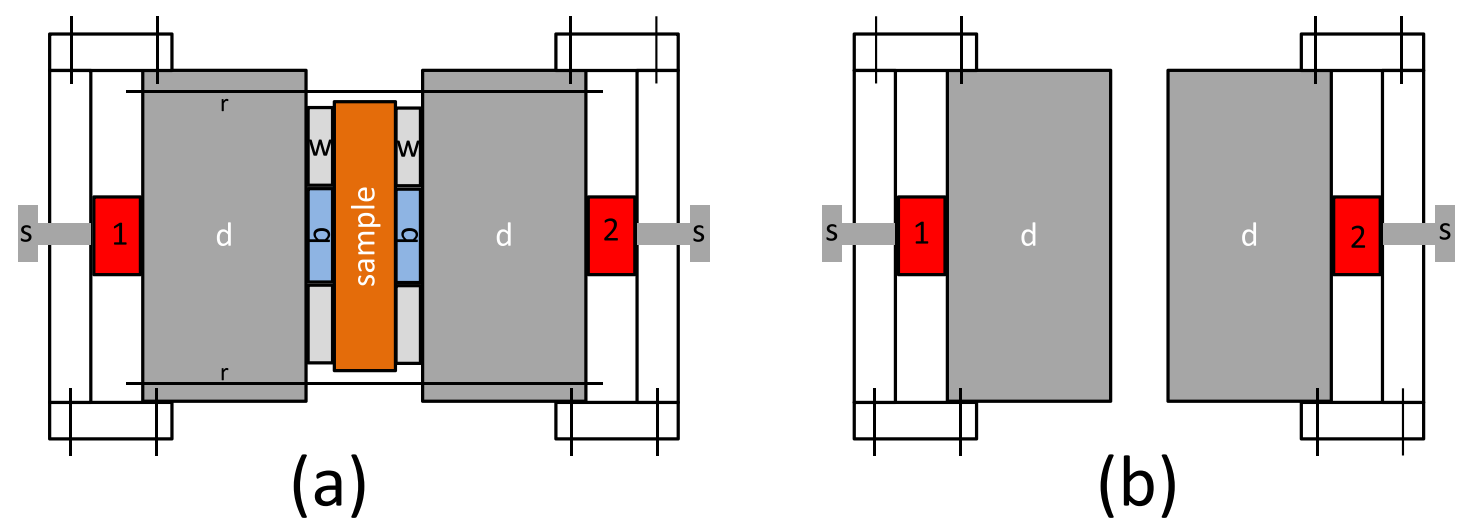

FIG. 1. (Color online) Sketch of the two different configurations of the experimental setup. 1, 2: shear wave transducers. d: delay lines. w: washers playing the role of spacers. b: viscoelastic bond. sample: slab of solid material of interest. s: screw tightening together the transducer and the delay line. r: three threaded rods tightening together the assembly. (a) Mounted setup used to measure the signals reflected and transmitted by the five-layers sandwich. (b) Unmounted setup used to measure the calibration signals. 


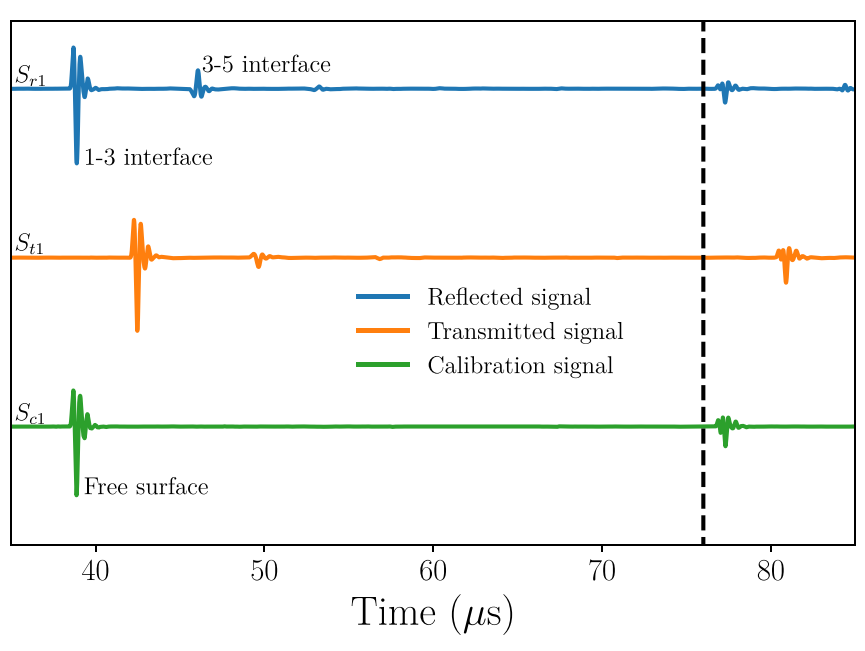

FIG. 2. (Color online) Reflection, transmission, and calibration signals corresponding to the $5 \mathrm{~mm}$ thick sample of PMMA and a pulse excitation at $f_{\mathrm{c}}=2.22 \mathrm{MHz}$ central frequency. The vertical dashed line indicates the end of signal analysis. Beyond this time, other echoes having experienced a reflection on one transducter-delay line interface are visible. Interface designation refers to labels defined in Fig. 4.

To that end, we interrupt the signal analysis at the instant shown as a vertical dashed line in Fig. 2, after which other echoes having experienced a reflection on one transducerdelay line interface are visible. Observation of Fig. 2 leads to the following remarks:

- Contrary to the calibration signal, the reflection and transmission signals display a series of grouped echoes that evidence the ringing of the shear wave inside the sample.

- The echoes have the same shape, which denotes weak dispersion.

- The variation of the polarity of the echoes of the reflection and transmission signals are the signature of the sign change of stress at reflection on the metal-PMMA effective interface and of the sign invariance of stress at reflection on the PMMA-metal effective interface.

\section{Determination of reflection and transmission coefficients}

Fourier transforms of reflection, transmission and calibration signals, called hereafter spectra, are then computed.

Since the signals are interrupted before the detection of echoes having experienced a reflection on one transducterdelay line, delay lines can be considered as semi-infinite media for signal analysis. Moreover, due to the far field hypothesis, propagation of plane waves is assumed in each layer.

In the following, $I(\omega)$ is the spectrum of the electric signal applied to the emitter $(\omega=2 \pi f$ is the angular frequency), $g_{\mathrm{d} m}$ the electro-mechanical transduction coefficient from transducer $m$ to the delay line, $m=1,2$, and $g_{m \mathrm{~d}}$ the electro-mechanical transduction coefficient from the delay line to transducer $m, m=1,2$ (cf. Fig. 1). Since signal analysis excludes echoes having experienced a reflection on one transducter-delay line interface, the spectra of calibration signals satisfy

$$
S_{\mathrm{c} m}(\omega)=-I(\omega) g_{m \mathrm{~d}} g_{\mathrm{d} m} e^{2 j k_{\mathrm{d}} L}, \quad m=1,2,
$$

where $k_{\mathrm{d}}=\omega / c_{\mathrm{d}}$ is the wave number in the delay lines. The spectra of reflection and tranmission signals satisfy

$$
\left\{\begin{array}{l}
S_{\mathrm{r} m}(\omega)=I(\omega) \mathcal{R} g_{m \mathrm{~d}} g_{\mathrm{d} m} e^{2 j k_{\mathrm{d}} L} \\
S_{\mathrm{t} m}(\omega)=I(\omega) \mathcal{T} g_{m \mathrm{~d}} g_{\mathrm{d} n} e^{2 j k_{\mathrm{d}} L}
\end{array}\right.
$$

where $n=2$ if $m=1$ and $n=1$ if $m=2, \mathcal{R}$ is the stress reflection coefficient of the bond-sample-bond assembly and $\mathcal{T}$ is its stress transmission coefficient assuming bond layers with identical thicknesses. Thus, $\mathcal{R}$ and $\mathcal{T}^{2}$ can be experimentally evaluated from the following expressions:

$$
\mathcal{R}_{m}=-\frac{S_{\mathrm{r} m}}{S_{\mathrm{c} m}}, \quad m=1,2 \quad \text { and } \quad \mathcal{T}^{2}=\frac{S_{t 1} S_{t 2}}{S_{c 1} S_{c 2}},
$$

where $\mathcal{R}_{1}$ and $\mathcal{R}_{2}$ are the two independent evaluations of $\mathcal{R}$ on each side of the setup.

Figure 3 displays the variation as a function of frequency of the magnitude and argument of $\mathcal{R}_{1}, \mathcal{R}_{2}$, and $\mathcal{T}^{2}$ determined experimentally using Eq. (1) for the $5 \mathrm{~mm}$ thick sample and a pulse excitation at $4.18 \mathrm{MHz}$ central frequency. Their quasi-periodic behavior observed over a broad frequency range of $0.5-8 \mathrm{MHz}$ is the signature of the multiple interferences of the ringing shear wave inside the bondsample-bond assembly. Note that $\mathcal{R}$ and $\mathcal{T}$ are not expected to satisfy $|\mathcal{R}|^{2}+|\mathcal{T}|^{2}=1$ because of dissipation in the bond-sample-bond assembly.

The frequency domain of validity of these experimental determinations of $\mathcal{R}$ and $\mathcal{T}^{2}$ can be determined according to several criteria that have to be simultaneously satisfied:
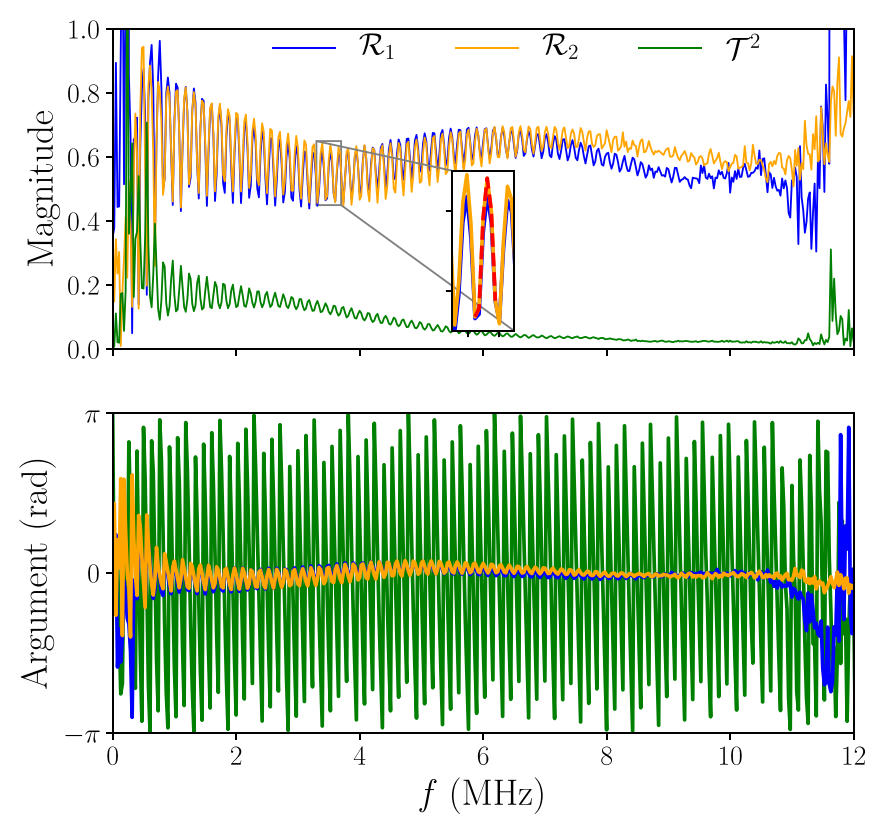

FIG. 3. (Color online) Variation as a function of frequency of the magnitude and argument of $\mathcal{R}_{1}$ and $\mathcal{R}_{2}$ and of the square of $\mathcal{T}$ determined experimentally using Eq. (1) for the $5 \mathrm{~mm}$ thick sample of PMMA and a pulse excitation at $f_{\mathrm{c}}=4.18 \mathrm{MHz}$ central frequency. Inset: zoom on the oscillations of $\mathcal{R}_{1}$ and $\mathcal{R}_{2}$. An example of portion of signal along which the averaged error function defined by Eq. (13) is calculated is highlighted as a red dashed curve. 
- In order for the waves propagating through the sample to be considered as plane waves, the sample has to be located in the far-field of the emitter. The corresponding upper boundary for frequency is $f=f_{\max }$ such that $z_{\mathrm{R}} \approx k_{\mathrm{d}} a^{2} / 2 \pi=L$ (Cheeke, 2002). $f_{\max }=4.7 \mathrm{MHz}$ for the transducers used in this study.

- In order to avoid any propagation through the brass spacers, the beam (main diffraction lobe) diameter has to be smaller than the washer internal diameter $D_{\mathrm{w}}$. The corresponding lower boundary for frequency is $f=f_{\min }$ such that (Cheeke, 2002)

$$
\arcsin \left(3.83 /\left(k_{\mathrm{d}} a\right)\right)=\arctan \left[\left(D_{\mathrm{w}} / 2-a\right) /\left(L-z_{\mathrm{R}}\right]\right) .
$$

Here, $f_{\min }=0.86 \mathrm{MHz}$ for the transducers used in this study.

- Since $\mathcal{R}_{1}, \mathcal{R}_{2}$ are two independent measurements of the same quantity $\mathcal{R}$, the less $\mathcal{R}_{1}$ and $\mathcal{R}_{2}$ coincide, the less the data are trustful; however, the relationship between the $\mathcal{R}_{1}-\mathcal{R}_{2}$ mismatch and the uncertainty on the sample shear modulus $G$ could not be quantified. $\mathcal{R}_{1}-\mathcal{R}_{2}$ mismatch between 7 and $9 \mathrm{MHz}$ could be ascribed to tridimensional characteristic features of the setup that show up at large frequencies such as defaults of planeity or parallelism of delay line-bond and bond-sample interfaces, or to slightly different bond thicknesses.

As shown in Fig. 3, $\mathcal{R}_{1}$ and $\mathcal{R}_{2}$ (i) coincide from $f_{\min }$ up to $7 \mathrm{MHz} \simeq 1.5 f_{\max }$, (ii) separate above $7 \mathrm{MHz}$. $\mathcal{R}_{1}$ and $\mathcal{R}_{2}$ look noisy below $0.3 \mathrm{MHz}$ and above $9 \mathrm{MHz}$ as the result of small signal-to-noise ratio far away from $f_{\mathrm{c}}$.

\section{RESOLUTION OF THE INVERSE PROBLEM}

In this section, we present the method used to determine the sample shear modulus from the measured reflection and transmission coefficients.

\section{A. Interference model}

Since the signals are interrupted before the detection of echoes having experienced a reflection on one transducerdelay line interface, delay lines can be considered as semiinfinite media. The one-dimensional geometry assumed in the model is shown in Fig. 4.

The properties of the 2017 A Dural alloy constituting both delay lines at $(5.0 \pm 0.2)^{\circ} \mathrm{C}$ are mass density $\rho_{\mathrm{d}}$ $=(2790 \pm 10) \mathrm{kg} \cdot \mathrm{m}^{-3}$ and shear wave velocity $c_{\mathrm{d}}=(3117$ $\pm 12) \mathrm{m} \cdot \mathrm{s}^{-1}$, determined by time of flight measurement on

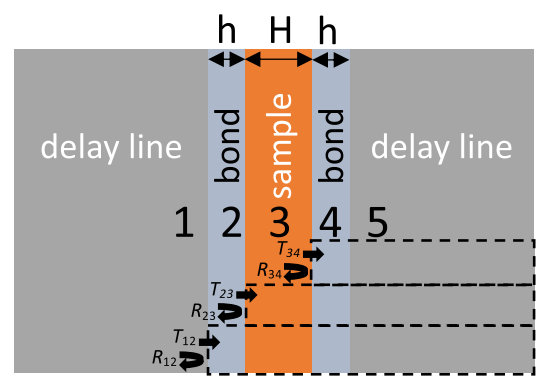

FIG. 4. (Color online) Sketch and notations for the interference model. calibration signals according to the procedure described in Appendix C. This corresponds to a shear modulus (considered as real) $G_{\mathrm{d}}=(27.1 \pm 0.2) \mathrm{GPa}$.

As detailed in Appendix A, the bond shear modulus $G_{\mathrm{b}}$ is complex and has been precisely measured in the investigated frequency domain. Bond mass density is $\rho_{\mathrm{b}}=(1452$ $\pm 27) \mathrm{kg} \cdot \mathrm{m}^{-3}$ at ambient temperature. PMMA mass density at $(5.0 \pm 0.2)^{\circ} \mathrm{C}$ is $\rho=(1187 \pm 4) \mathrm{kg} \cdot \mathrm{m}^{-3}$.

For the purpose of the following demonstration, the (possibly complex) wavenumbers in the five-layers shown in Fig. 4 are defined as

$$
k_{i}=\omega \sqrt{\frac{\rho_{i}}{G_{i}}}, \quad i=1 \text { to } 5,
$$

where $\rho_{i}$ is the mass density of layer $i$ and $G_{i}$ its possibly complex shear modulus.

The reflection and transmission coefficients of the bondsample-bond assembly are obtained by an iterative method. First, we express the stress reflection coefficient $r_{n m}$ and the stress transmission coefficient $t_{n m}$ of an interface between two solids labeled $n$ and $m$ when the incident shear wave propagates in solid $n$

$$
\left\{\begin{array}{l}
r_{n m}=\frac{\rho_{m} k_{n}-\rho_{n} k_{m}}{\rho_{m} k_{n}+\rho_{n} k_{m}}, \\
t_{n m}=\frac{2 \rho_{m} k_{n}}{\rho_{m} k_{n}+\rho_{n} k_{m}} .
\end{array}\right.
$$

According to Fig. 4, layers 1 and 5 have identical properties, the same for layers 2 and 4 . It follows that:

$$
\left\{\begin{array}{l}
r_{12}=r_{54}=-r_{21}=-r_{45}, \\
t_{12}=t_{54}, \\
t_{21}=t_{45}, \\
r_{23}=r_{43}=-r_{32}=-r_{34}, \\
t_{23}=t_{43}, \\
t_{32}=t_{34}
\end{array}\right.
$$

The wave reflected by the interface between layer 1 and the $2-3-4-5$ assembly (associated reflection coefficient $\mathcal{R}$ $=R_{12}$, see Fig. 4) is the superposition of the waves reflected by the $1-2$ interface (associated reflection coefficient $r_{12}$ ) and by the interface between layer 2 and the $3-4-5$ assembly (associated reflection coefficient $R_{23}$, see Fig. 4). The reflection coefficient $\mathcal{R}$ satisfies

$$
\mathcal{R}=R_{12}=r_{12}+\frac{t_{12} R_{23} t_{21} e^{2 j k_{2} h}}{1+r_{12} R_{23} e^{2 j k_{2} h}} .
$$

In the same manner, $R_{23}$ satisfies

$$
R_{23}=r_{23}+\frac{t_{23} R_{34} t_{32} e^{2 j k_{3} H}}{1+r_{23} R_{34} e^{2 j k_{3} H}},
$$

and $R_{34}$ is given by

$$
R_{34}=-r_{23}-\frac{t_{32} r_{12} t_{23} e^{2 j k_{2} h}}{1+r_{23} r_{12} e^{2 j k_{2} h}} .
$$


Employing the same procedure, we express the transmission coefficient of the interface between layer 1 and the $2-3-$ $4-5$ assembly $\mathcal{T}=T_{12}$ as

$$
\mathcal{T}=T_{12}=\frac{t_{12} T_{23} e^{j k_{2} h}}{1+r_{12} R_{23} e^{2 j k_{2} h}},
$$

where $T_{23}$ is given by

$$
T_{23}=\frac{t_{23} T_{34} e^{j k_{3} H}}{1+r_{23} R_{34} e^{2 j k_{3} H}},
$$

with

$$
T_{34}=\frac{t_{32} t_{21} e^{j k_{2} h}}{1+r_{23} r_{12} e^{2 j k_{2} h}} .
$$

Equations (3)-(10) can be used to determine the sample shear modulus $G_{3}=G$ from the measured $\mathcal{R}$ and $\mathcal{T}$. We call this the inverse problem. In the next section, we present the methods developed to solve it.

\section{B. Resolution of the inverse problem}

We are seeking the frequency-dependent complex shear modulus of the sample material

$$
G(\omega)=G^{\prime}(\omega)-j G^{\prime \prime}(\omega)
$$

that (i) results in reflection and transmission coefficients $\mathcal{R}$ and $\mathcal{T}$ as close as possible to the measured ones $\left(G^{\prime}\right.$ and $G^{\prime \prime}$ are real functions of $\omega$ ) and (ii) is physically relevant. To this aim, for each discrete value $\omega_{i}$ of the angular frequency at which $\mathcal{R}$ and $\mathcal{T}$ have been measured, we aim to minimize with respect to $G^{\prime}\left(\omega_{i}\right)$ and $G^{\prime \prime}\left(\omega_{i}\right)$ the error function

$$
\varepsilon_{i}=\left|R_{12}\left(G\left(\omega_{i}\right)\right)-\mathcal{R}_{2}\left(\omega_{i}\right)\right|^{2}+\left|T_{12}^{2}\left(G\left(\omega_{i}\right)\right)-\mathcal{T}^{2}\left(\omega_{i}\right)\right|^{2},
$$

where $R_{12}\left(G\left(\omega_{i}\right)\right)$ is defined by Eqs. (3) and (5)-(7), $T_{12}\left(G\left(\omega_{i}\right)\right)$ by Eqs. (3), and (6)-(10) and $\mathcal{R}_{1}$ and $\mathcal{T}^{2}$ by Eq. (1). Since $\mathcal{R}$ and $\mathcal{T}$ are quasi-periodic functions of $k_{3} H$ (they would be strictly periodic in absence of dissipation and in the $h \ll H$ limit), at each frequency $G$ is expected to be multi-valued. To univocally determine $G$, we implemented a procedure presented in detail thereafter.

Technically, since $\mathcal{R}$ and $\mathcal{T}$ oscillate with $\omega$, in order to smooth the variations of $G^{\prime}$ and $G^{\prime \prime}$ with $\omega$, we rather minimize the cumulative sum of errors over one period of their oscillation (encompassing $2 N+1$ samples), as illustrated in the inset of Fig. 3

$$
\bar{\varepsilon}_{i}=\sum_{q=i-N}^{i+N} \varepsilon_{q}
$$

The variation of $\bar{\varepsilon}$ at $f=f_{\mathrm{c}}$ as function of $G^{\prime}$ and $G^{\prime \prime}$ is displayed around its absolute minimum in Fig. 5(a) for the $5 \mathrm{~mm}$ thick sample and a pulse excitation at $f_{\mathrm{c}}=2.22 \mathrm{MHz}$. As expected, $\bar{\varepsilon}$ exhibits several minima in the $\left(G^{\prime}, G^{\prime \prime}\right)$ plane.
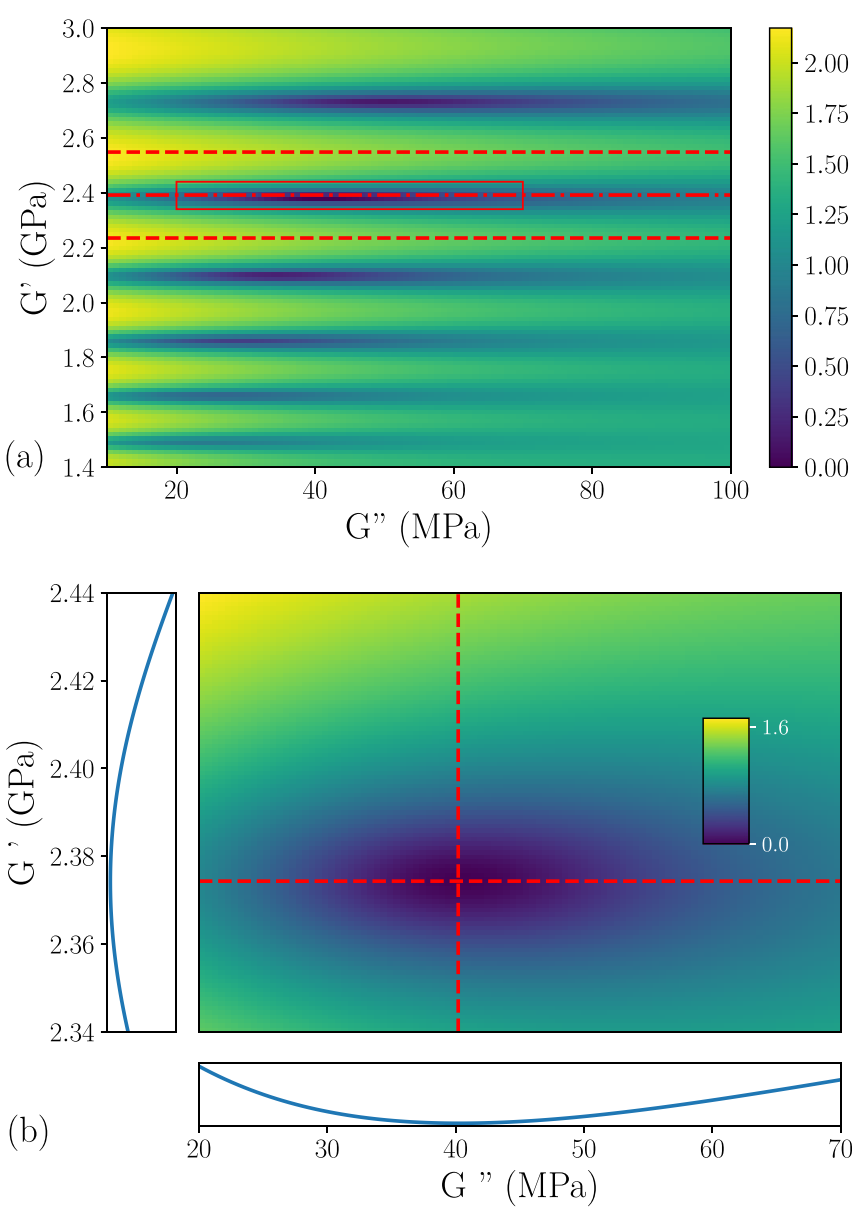

FIG. 5. (Color online) (a) Variations in color scale of the decadic logarithm of the relative averaged error function $\log [\bar{\varepsilon} / \min (\bar{\varepsilon})]$ in the vicinity of its absolute minimum $\min (\bar{\varepsilon})$ at $f=\omega / 2 \pi=2.22 \mathrm{MHz}$ as function of the real and imaginary parts $G^{\prime}$ and $G^{\prime \prime}$ of the sample complex shear modulus $G$ for the $5 \mathrm{~mm}$ thick sample and a pulse excitation at $f_{\mathrm{c}}=2.22 \mathrm{MHz}$ central frequency. The red dot-dashed line indicates the value of $G^{\prime}$ deduced from time of flight measurement (see text) and the dashed lines its uncertainty. The red solid frame indicates the enlarged area shown below. (b) Enlarged area. The red dashed lines indicate the values of $G^{\prime}$ and $G^{\prime \prime}$ for which $\bar{\varepsilon}$ is minimum. Graphs below and on the left: variations of $\bar{\varepsilon}$ versus $G^{\prime}$ and $G^{\prime \prime}$ in linear scales along the red dashed lines.

These minima moving continuously with frequency in the $\left(G^{\prime}, G^{\prime \prime}\right)$ plane, we are left with several branches of solutions in the $\left(f, G^{\prime}\right)$ and $\left(f, G^{\prime \prime}\right)$ planes. Three branches labelled $A, A^{\prime}$, and $A^{\prime \prime}$ are displayed in Figs. 6(a) and 6(b). To identify the physically relevant branch of solutions, we have implemented two strategies that we present one after the other: time of flight analysis and the use of samples with different thicknesses.

\section{Time of flight analysis}

Here we demonstrate how time of flight measurement allows us to evaluate $G^{\prime}$ at central frequency $f_{\mathrm{c}}$ and thus helps us identifying the physically relevant branch of solutions.

We measure the laps of time $\tau$ between the first and the second reflected echoes (see Fig. 2) that roughly corresponds to the duration of a round trip between 1-2 and 4-5 interfaces (see Fig. 4) given their large impedance contrasts. From 

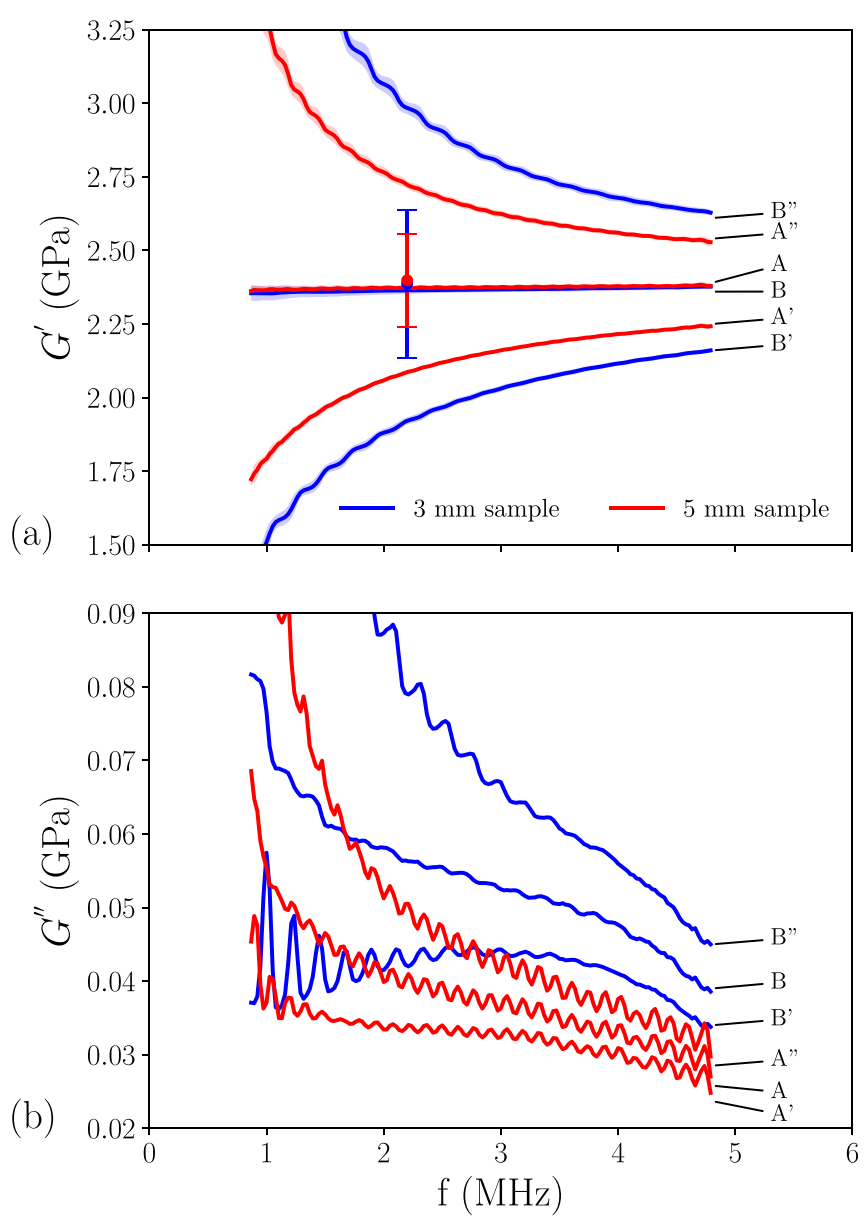

FIG. 6. (Color online) Branches of solutions to the error minimization problem (a) in the $\left(f, G^{\prime}\right)$ plane, (b) in the $\left(f, G^{\prime \prime}\right)$ plane. $A, A^{\prime}, A^{\prime \prime}$ branches correspond to the $5 \mathrm{~mm}$ thick sample and a pulse excitation at $f_{\mathrm{c}}=2.22 \mathrm{MHz}$. $B, B^{\prime}, B^{\prime \prime}$ branches correspond to the $3 \mathrm{~mm}$ thick sample and a pulse excitation at $f_{\mathrm{c}}=2.22 \mathrm{MHz}$. Errobars in (b) are omitted for clarity purpose. Dot symbols supplied with errorbars: value of $G^{\prime}$ determined at $f_{\mathrm{c}}$ from time of flight measurement.

$$
\tau=\frac{4 h}{c_{\mathrm{b}}}+\frac{2 H}{c},
$$

we evaluate the shear wave group velocity in the sample $c$. Then, we evaluate the sample shear modulus $G^{\prime}$ using $G^{\prime}$ $\simeq \rho c^{2}$ since $G^{\prime \prime} \ll G^{\prime}$ (see Fig. 6). Taking account of the uncertainties on $\tau, h, H$ and $c_{\mathrm{b}}$, we find $G^{\prime}=(2.40 \pm 0.16)$ $\mathrm{GPa}$; see Appendix C for details.

As shown in Fig. 5(a), the value of $G^{\prime}$ deduced from time of flight measurement coincide within experimental uncertainty with one minimum of $\bar{\varepsilon}$, namely its absolute minimum at $f=f_{\mathrm{c}}$. This allows us to identify the physically relevant value of $G^{\prime}$, hence of $G^{\prime \prime}$ at $f=f_{\mathrm{c}}$. Continuity of $G(\omega)$ allows us to deduce the physically relevant branch of solutions in the $\left(f, G^{\prime}\right)$ and $\left(f, G^{\prime \prime}\right)$ planes [branch $A$ in Figs. 6(a) and 6(b)]. The variations as function of frequency of $R_{12}$ and $T_{12}^{2}$ computed using the values of $G$ encountered along branch $A$ are displayed together with the measured $\mathcal{R}_{1}, \mathcal{R}_{2}$, and $\mathcal{T}^{2}$ in Figs. 7(a) and 7(b). The coincidence between $R_{12}, \mathcal{R}_{1}$, and $\mathcal{R}_{2}$ and between $T_{12}^{2}$ and $\mathcal{T}^{2}$ in the frequency range at which signals are not noisy demonstrates the accuracy of the resolution of the inverse problem. This accuracy is more quantitatively

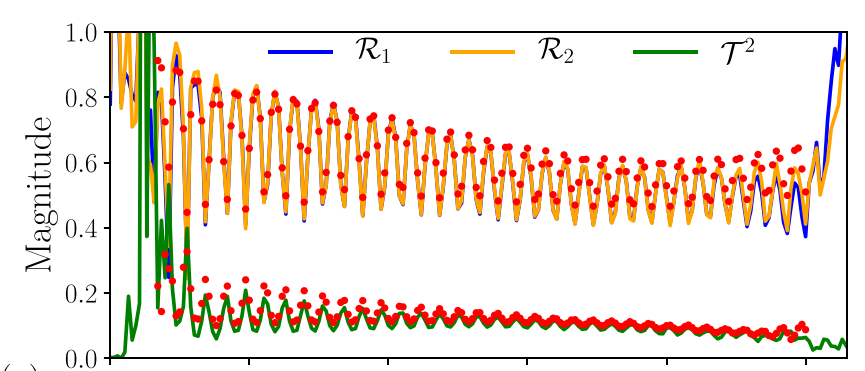

(a)
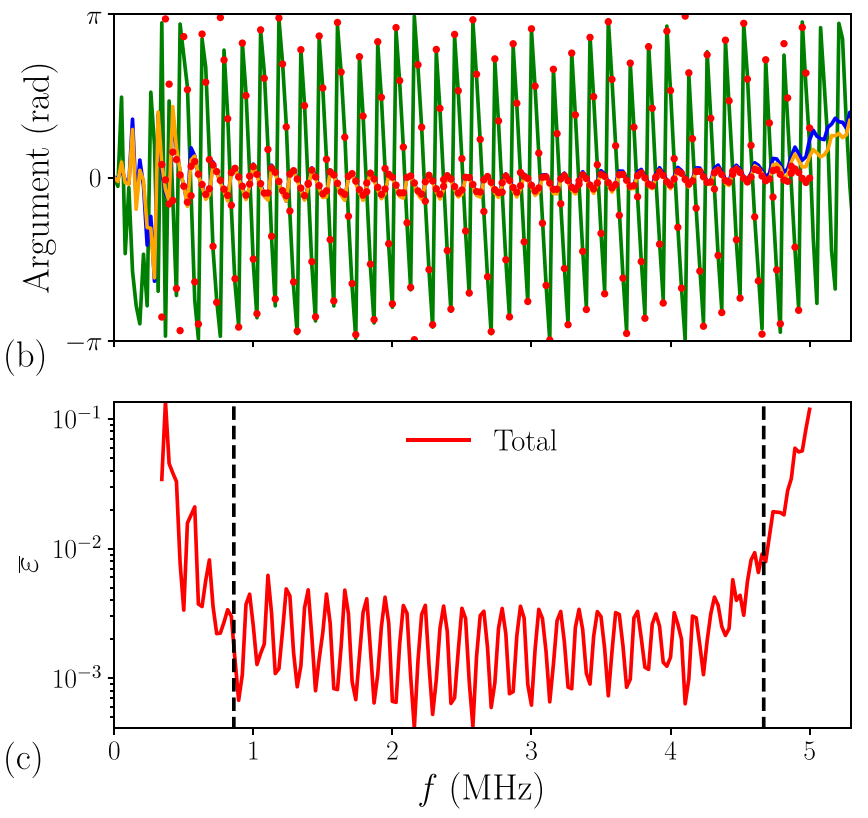

FIG. 7. (Color online) Variations vs frequency of (a) the magnitude and (b) the argument of the measured reflection coefficients $\mathcal{R}_{1}$ (blue curve), $\mathcal{R}_{2}$ (orange curve), and squared transmission coefficient $\mathcal{T}^{2}$ (green curve) defined by Eq. (1) for the $5 \mathrm{~mm}$ thick sample and a pulse excitation at $f_{\mathrm{c}}$ $=2.22 \mathrm{MHz}$ central frequency. Red symbols: variations vs frequency of $R_{12}$ and $T_{12}^{2}$ defined by Eqs. (5) and (8) and computed using the values of $G$ encountered along the branch of solutions $A$ (see Fig. 6). (c) Variation vs frequency of the residual error $\min (\bar{\varepsilon})$ along this branch.

assessed by considering the variation with frequency of $\bar{\varepsilon}$ evaluated along the physically relevant branch, displayed in Fig. 7(c). The lower $\bar{\varepsilon}$, the more accurately the plane wave interference model describes the behavior of the five-layers sandwich, so the more trustful is the determination of $G$. We note that $\bar{\varepsilon}$ takes low values (between $10^{-3}$ and $10^{-2}$ ) precisely in the frequency range $\left[f_{\min }, f_{\max }\right]$ and conversely increases outside of this range, which demonstrates the accuracy of the resolution of the inverse problem and the relevance of the definitions of $f_{\min }$ and $f_{\max }$ that delimit the domain of validity of the plane wave model.

This achieves the demonstration that time of flight analysis allows us to univocally determine $G$ in the case of small dispersion. Finally, we stress that the local minimum of $\bar{\varepsilon}$ followed by the physically relevant branch does not necessarily coincide at each frequency with the absolute minimum of $\bar{\varepsilon}$.

\section{Use of samples with different thicknesses}

Here we demonstrate how the use of samples with different thicknesses allows us to identify the physically 
relevant branch of solutions. With this aim in view, we probe the $3 \mathrm{~mm}$ thick sample using excitations at $f_{\mathrm{c}}=2.22 \mathrm{MHz}$ central frequency. Three branches of solutions $B, B^{\prime}$, and $B^{\prime \prime}$ are plotted in the $\left(f, G^{\prime}\right)$ plane in Fig. 6(a). Time of flight analysis leads to select branch $B$ as the physically relevant branch of solutions. Interestingly, we observe that branches $A$ and $B$ form a thin bundle, whereas at low frequency branch $B^{\prime}$ clearly separates from branches $A^{\prime}$ and branch $B^{\prime \prime}$ separates from branch $A^{\prime \prime}$. Since $G^{\prime}$ does not depend of course on the sample thickness, this confirms that the physically relevant branches of solutions are $A$ and $B$. This demonstrates that $G$ can be univocally determined by determining the branches of solutions that are invariant with respect to sample thickness changes. This criterium can be used in situations where time of flight analysis fails because of large dispersion or absorption.

We note that the resolution of this inverse problem has already been addressed in the past. (Fokin et al., 2007) theoretically proposed to identify the physically relevant branch of solutions by reducing the sample thickness to values smaller than $\lambda / 2$, where $\lambda$ is the wavelength at central frequency in the sample. Pierre et al. (2013) selected the physically relevant branch of solutions by comparison with trustworthy models of material rheology. The method we proposed is twofold, independent of any rheological model and was experimentally validated.

\section{PMMA RHEOLOGY}

The whole set of our measurements of $G^{\prime}(f)$ and $G^{\prime \prime}(f)$ performed using two different types of transducers and two samples with different thicknesses are shown in Fig. 8. Despite experimental uncertainties (see Appendix B), our measurements evidence dispersion in PMMA, more precisely a weak increase of $G^{\prime}$ and a weak decrease of $G^{\prime \prime}$ as frequency increases.

For the purpose of helping future readers to exploit our measurements, we provide a simple analytical representation valid in the frequency range $0.86-7 \mathrm{MHz}$ of our measurements of $G$ on the $3 \mathrm{~mm}$ thick sample using a pulse excitation at $f_{\mathrm{c}}=4.18 \mathrm{MHz}$ central frequency

$$
\left\{\begin{array}{l}
G^{\prime}(\text { in } \mathrm{GPa})=2.34+5.76 \times 10^{-3} f(\text { in } \mathrm{MHz}), \\
G^{\prime \prime}(\text { in } \mathrm{MPa})=42.3-2.05 f(\text { in } \mathrm{MHz}) .
\end{array}\right.
$$

Ultrasonic measurements of PMMA shear modulus at ambiant temperature have been previously reported in (Asay et al., 1969; Biwa et al., 2001). We note that no evaluation of the uncertainty on $G^{\prime}$ and $G^{\prime \prime}$ was provided. Thanks to the study of the temperature dependence of $G^{\prime}$ reported in Asay et al. (1969), we extrapolated the value of $G^{\prime}$ down to $5^{\circ}$. As shown in Fig. 8, our measurements of $G^{\prime}$ are in quantitative agreement with data of literature by less than 5\%. Our measurements of $G^{\prime \prime}$ agree with those of Asay et al. (1969), but not with those of Biwa et al. (2001), although the decreasing behavior of $G^{\prime \prime}$ observed in Biwa et al. is recovered.

The corresponding variations versus $f$ of the PMMA shear velocity
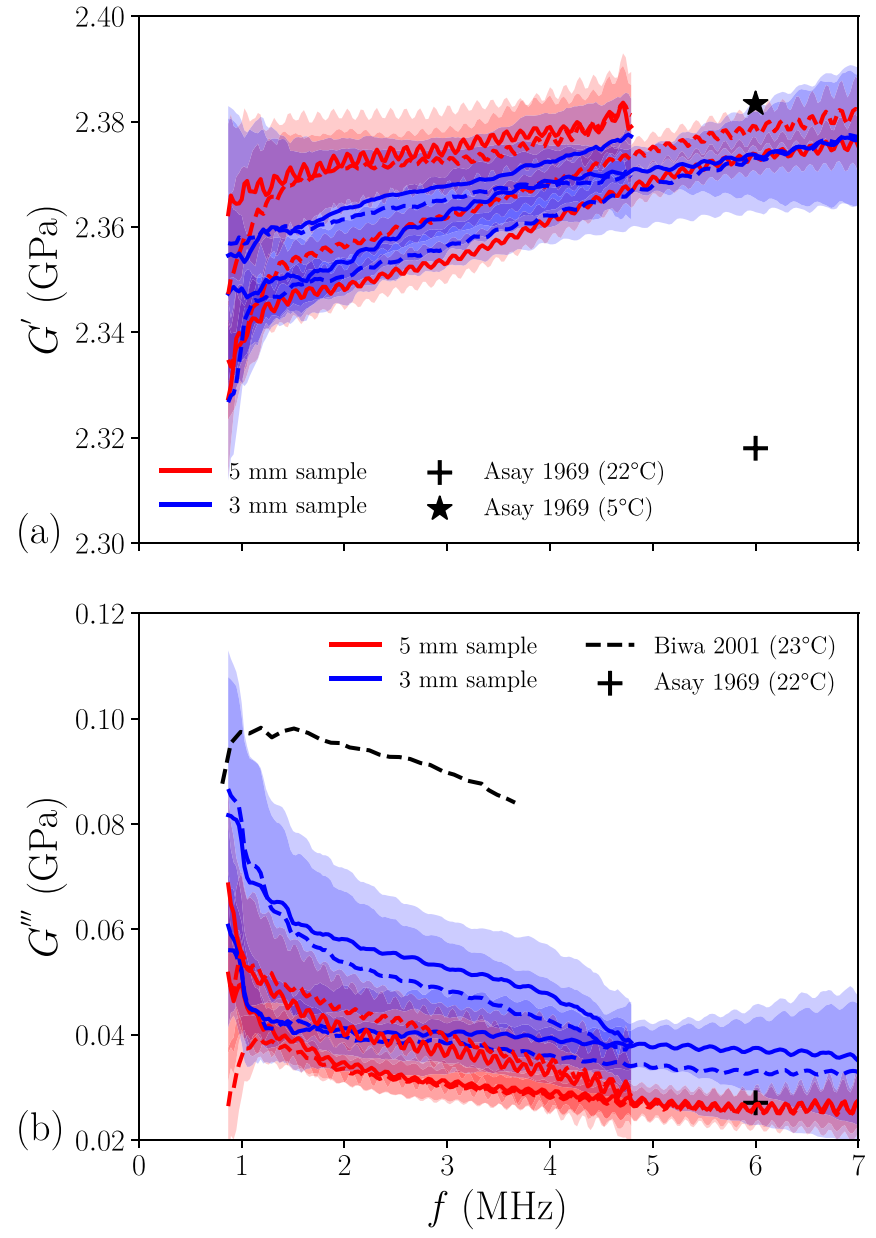

FIG. 8. (Color online) PMMA shear modulus: comparison with literature. Each pair of neighbouring (solid and dashed) curves of the same color correspond to two realizations in the same experimental conditions (same sample, same pair of transducers). The uncertainty associated to the determination of $G^{\prime}$ and $G^{\prime \prime}$ by error minimisation is represented as an extra thickness in light colors on both sides of the solid curves (see Appendix B 1). Biwa et al. (2001) measured $G^{\prime}=2.25 \mathrm{GPa}$ in the frequency range $2-5 \mathrm{MHz}$ at $23^{\circ} \mathrm{C}$.

$$
c=\sqrt{\frac{2|G|^{2}}{\rho\left(G^{\prime}+|G|\right)}},
$$

and attenuation

$$
\alpha=\frac{G^{\prime \prime} k}{G^{\prime}+|G|},
$$

where $k=k_{3}=\omega / c$, are plotted in Fig. 9 .

\section{CONCLUSION}

We have developed a new technique based on interferometry for measuring the complex shear modulus of thin slabs of viscoelastic solids over a broad frequency range. Thanks to thickness-controlled bonding, its reproducibility was found to be better than the uncertainty associated to a single measurement. Its overall accuracy was found to be principally limited by the uncertainty on the shear modulus of the bond. Nevertheless, the accuracy of this technique allowed us to reveal the dispersion of shear waves in PMMA in the range 1-7 MHz. Identification of a bond with high real 

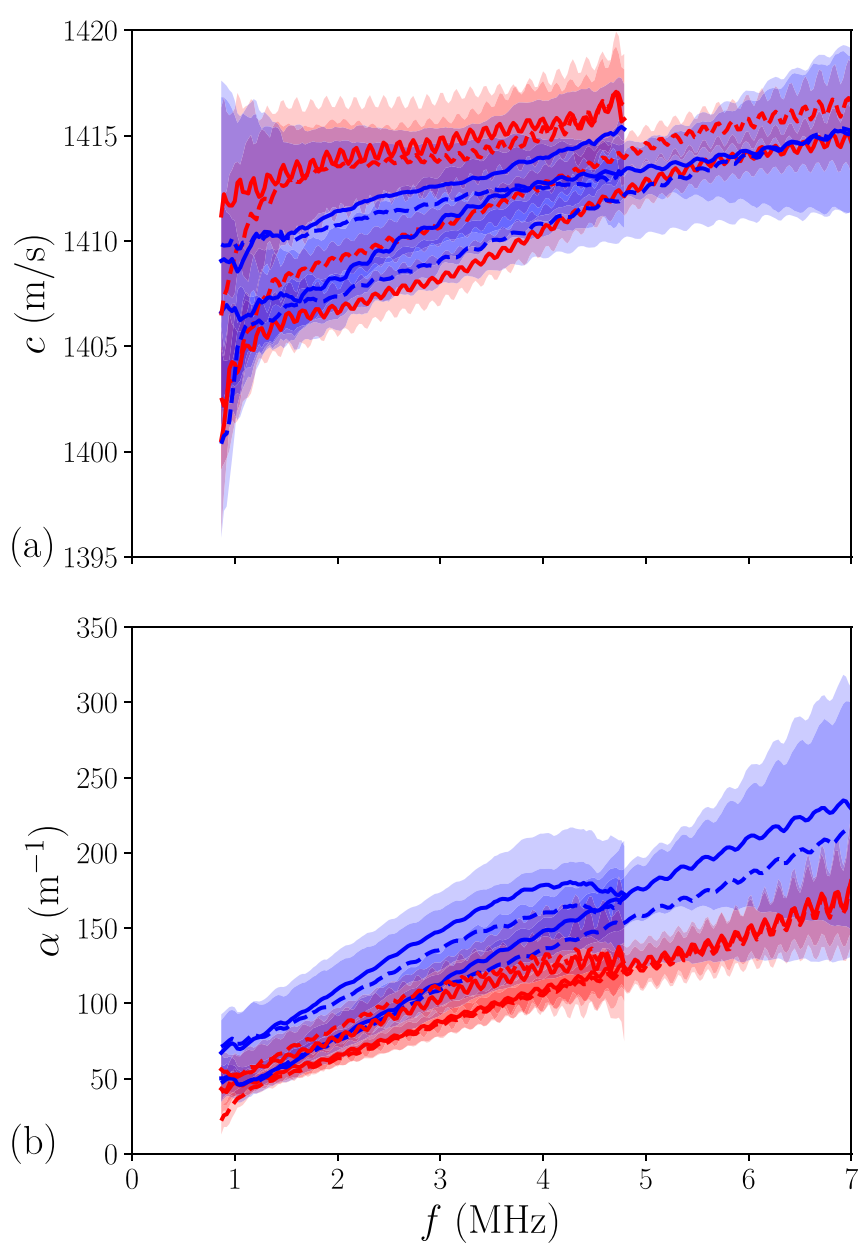

FIG. 9. (Color online) PMMA shear velocity $c$ (a) and attenuation $\alpha$ (b). Each pair of neighbouring (solid and dashed) curves of the same color correspond to two realizations in the same experimental conditions (same sample, same pair of transducers). The uncertainty associated to the determination of $c$ and $\alpha$ by error minimisation is represented as an extra thickness in light colors on both sides of the solid curves (see Appendix B 1).

shear modulus and usable at room temperature would enable to apply this technique at room temperature.

This technique should be especially suited to the measurement of the complex shear modulus of solid materials displaying noticeable attenuation. Soft solids, defined as those undergoing large deformations in the mounted fivelayers sandwich, should rather be studied using the threelayers sandwich described in Lefebvre et al. (2018) or reflection measurement at grazing incidence (Longin et al., 1998).

\section{APPENDIX A: BOND RHEOLOGY}

Bond rheology was determined using the three-layers sandwich setup and the procedure described in Lefebvre et al. (2018). The complex bond shear modulus $G_{\mathrm{b}}$ was extracted from ultrasonic measurements using the procedure described in Sec. III B. The uncertainties on $G_{\mathrm{b}}^{\prime}$ and $G_{\mathrm{b}}^{\prime \prime}$ were evaluated using the procedure described in Appendix B 1.

The bond shear modulus $G_{\mathrm{b}}$ was modeled using the following fractional derivative rheological model (Bagley and Torvik, 1983)

$$
G_{\mathrm{b}}=\frac{G_{\mathrm{b}, 0}+G_{\mathrm{b}, \infty}\left(j \omega \tau_{\mathrm{b}}\right)^{\alpha_{\mathrm{b}}}}{1+\left(j \omega \tau_{\mathrm{b}}\right)^{\alpha_{\mathrm{b}}}}
$$

where $G_{\mathrm{b} 0}$ is the static value of $G_{\mathrm{b}}, G_{\mathrm{b} \infty}$ a constant, $\tau_{\mathrm{b}}$ a relaxation time, and $\alpha_{b}$ the exponent associated to the fractional derivative model, which allows to spread the influence of the relaxation time. Such a model is more versatile than those involving continuous or discrete distributions of relaxation times. The best fit of the experimental data by Eq. (A1) determined by error minimization is obtained with $G_{\mathrm{b}, 0}=(1.96 \pm 0.06) \mathrm{GPa}, G_{\mathrm{b}, \infty}=(3.58 \pm 0.04) \mathrm{GPa}$, $\tau_{\mathrm{b}}=(131 \pm 11) \mathrm{ns}, \alpha_{\mathrm{b}}=0.67 \pm 0.03$ (uncertainties were evaluated using the procedure described in Appendix B 1). This model for $G_{\mathrm{b}}$ was used in the interference model presented in Sec. III A.

To measure the bond mass density, we used a pycnometer and mineral oil with relative density $(0.850 \pm 0.002)$ as reference liquid (mineral oil for spectroscopy, CAS number 8042-47-5 from ACROS Organics). Bond mass density at ambiant temperature $\left(19^{\circ} \mathrm{C}\right)$ is $\rho_{\mathrm{b}}=(1452 \pm 27) \mathrm{kg} \cdot \mathrm{m}^{-3}$.

\section{APPENDIX B: EXPERIMENTAL UNCERTAINTIES}

The ultimate uncertainty on the sample shear modulus $G$ results from the combination of several sources of uncertainty: propagation of the uncertainties on the numerous parameters entering the interference model, uncertainty associated to the procedure of minimization of the error function $\bar{\varepsilon}$, and reproducibility. We review these various sources of uncertainty.

\section{Error minimization}

First, we quantify the uncertainty associated to the minimization of the error function $\bar{\varepsilon}$ defined by Eq. (13). As shown in Fig. 5(b) that is an enlargement of Fig. 5(a), the variations of $\bar{\varepsilon}$ in the vicinity of its absolute minimum are quadratic with $G^{\prime}$ and $G^{\prime \prime}$. Thus, we define the characteristic value of the uncertainty on $G^{\prime}$ associated to error minimization as the half of the interval of values of $G^{\prime}$ along which $\bar{\varepsilon}$ is larger than its minimum by less than $3 \mathrm{~dB}$. To do this, we determine the best fit of the variations of $\bar{\varepsilon}$ vs $G^{\prime}$ by the polynomial form: $\bar{\varepsilon}=\min (\bar{\varepsilon})\left[1+\left(G^{\prime}-G_{0}^{\prime}\right)^{2} / \delta^{2}\right]$, where $G_{0}^{\prime}$ corresponds to the minimum of $\bar{\varepsilon}$. The uncertainty on $G^{\prime}$ is thus $\Delta_{1} G^{\prime}=\sqrt{\sqrt{2}-1} \delta \simeq 0.64 \delta$ (same definition of uncertainty on $\left.G^{\prime \prime}\right)$. This uncertainty is represented in Figs. 8 and 10 as an extra thickness in light colors on both sides of the solid curves.

As shown in Fig. 8, the relative uncertainty on $G^{\prime \prime}$ is much larger than the one on $G^{\prime}$, as the consequence of $\bar{\varepsilon}$ minima much more spread along the $G^{\prime \prime}$ direction than along the $G^{\prime}$ direction; see Fig. 5.

\section{Reproducibility}

To test the reproducibility of the experiment, for each set of experimental conditions (given sample, given pair of transducers), the whole experimental procedure (starting from the unmounted setup at ambient temperature) has been repeated several times and two results have been systematically reported in Figs. 8 and 9 as solid and dashed curves of the same color. We observe that the gap between two realizations is smaller 

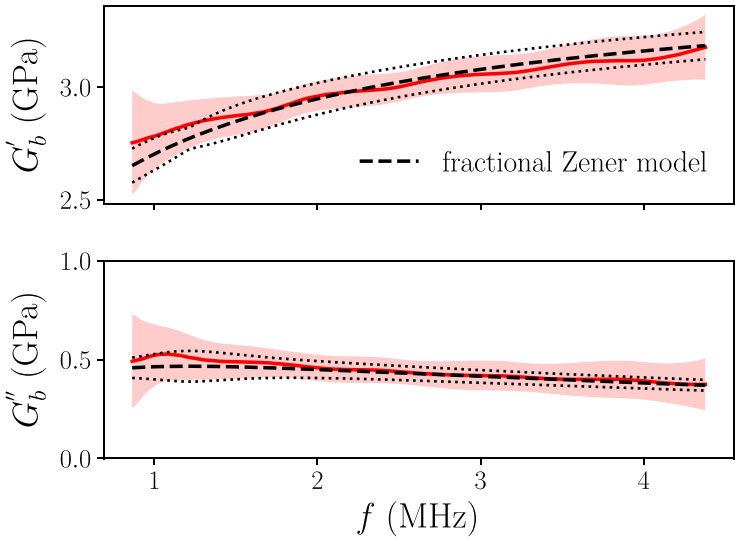

FIG. 10. (Color online) Bond complex shear modulus $G_{\mathrm{b}}$. Red solid curve with excess thickness in light color: measurements with their uncertainty as defined in Appendix B 1. Black dashed curve: best fit by the fractional Zener model. The black dotted curves are the uncertainty boundaries determined from the uncertainties on the parameters of the rheological model [Eq. (A1)].

than the uncertainty associated to error minimization for both $G^{\prime}$ and $G^{\prime \prime}$. This evidences the excellent reproducibility of our experiments, which usually constitutes a hard issue of experiments involving shear waves. We believe that the remaining factors that limit the reproducibility are (i) the absorption of water by the bond during the sandwich assembly that depends on the ambient air humidity and temperature, and (ii) the emission of parasitic longitudinal waves by the shear wave transducers that depends on the model of transducers and their conversion to shear waves at the delay line ends due to possible defects of parallelism.

\section{Propagation of uncertainties}

As shown in Sec. III A, the reflection and transmission coefficients $R_{12}$ and $T_{12}$ that are fitted to the measured coefficients $\mathcal{R}$ and $\mathcal{T}$ depend not only on $G(\omega)$ but also on the parameters $\rho_{\mathrm{d}}, c_{\mathrm{d}}, \rho_{\mathrm{b}}, G_{\mathrm{b}}(\omega), h, \rho$, and $H$. Thus, the uncertainties on these parameters result in a total uncertainty $\Delta_{2} G$ that adds to $\Delta_{1} G$. In order to estimate $\Delta_{2} G$ and to identify the main sources of uncertainty, we computed the uncertainty on $G^{\prime}$ at $f=2.22 \mathrm{MHz}$ and for a $5 \mathrm{~mm}$ thick sample, $\Delta_{x} G^{\prime}$, resulting from the uncertainty on $x, \Delta x$, as $\Delta_{x} G^{\prime}=\left|\left(\partial G^{\prime} / \partial x\right)\right| \Delta x, x$ standing for $\rho_{\mathrm{d}}, c_{\mathrm{d}}, \rho_{\mathrm{b}}, G_{\mathrm{b}}^{\prime}, G_{\mathrm{b}}^{\prime \prime}, h$, $\rho$, and $H$ (the same for $G^{\prime \prime}$ ). The resulting relative uncertainties are given in Table I. Note that the main source of uncertainty on $G$ is the uncertainty on the couplant shear modulus $G_{\mathrm{b}}$ and that the associated uncertainty on $G^{\prime \prime}$ is comparable to $\Delta_{1} G^{\prime \prime}$. On the contrary, the other sources of uncertainty

TABLE I. Relative uncertainties on $G$ resulting from the uncertainties on the experimental parameters.

Parameter $x$

\begin{tabular}{lcccccccc}
\cline { 2 - 8 } $\begin{array}{l}\text { Relative } \\
\text { uncertainty (\%) }\end{array}$ & $\rho_{\mathrm{d}}$ & $c_{\mathrm{d}}$ & $\rho_{\mathrm{b}}$ & $G_{\mathrm{b}}^{\prime}$ & $G_{\mathrm{b}}^{\prime \prime}$ & $h$ & $\rho$ & $H$ \\
\hline$\Delta_{x} G^{\prime} / G^{\prime}$ & 0.002 & 0.002 & 0.05 & 0.1 & 0.02 & 0.1 & 0.4 & 0.2 \\
$\Delta_{x} G^{\prime \prime} / G^{\prime \prime}$ & 0.4 & 0.4 & $\mathbf{2}$ & $\mathbf{2}$ & $\mathbf{5}$ & 0.1 & 0.4 & 0.2 \\
\hline \hline
\end{tabular}

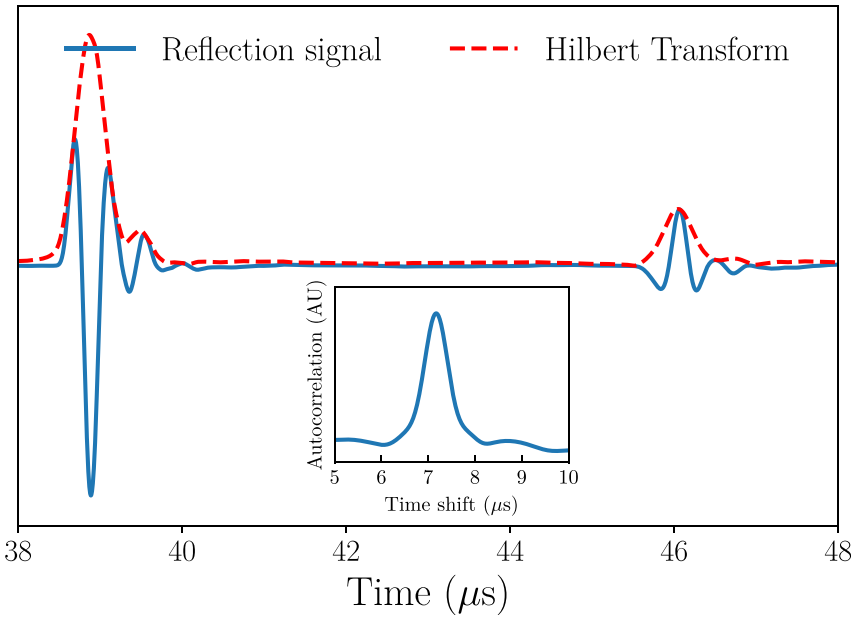

FIG. 11. (Color online) Zoom on the reflection signal (solid blue curve) corresponding to a $5 \mathrm{~mm}$ thick sample of PMMA and a pulse excitation at $f_{\mathrm{c}}=2.22 \mathrm{MHz}$ central frequency. Red dashed curve: Hilbert transform. Inset: Autocorrelation of the Hilbert transform of the reflection signal.

result in insignificant uncertainties compared to $\Delta_{1} G$. The impact of the uncertainty on $G_{\mathrm{b}}$ is expected to be smaller in the case of more absorbing materials.

\section{APPENDIX C: TIME OF FLIGHT MEASUREMENTS}

Problem inversion can be achieved by time of flight measurements (see Sec. III B 1) that require technical details given here. The laps of time $\tau$ between the first and the second reflected echoes visible in Fig. 2 of a pulse excitation at central frequency $f_{\mathrm{c}}=2.22 \mathrm{MHz}$ is measured by computing first the Hilbert transform of the reflection signal, shown in Fig. 11, then its autocorrelation, shown in inset of Fig. 11. $\tau$ is defined as the time shift corresponding to the maximum of the peak of the autocorrelation signal. Its uncertainty is defined as half of the peak width at $-3 \mathrm{~dB}$. We find $\tau=(7.17 \pm 0.23) \mu \mathrm{s}$.

The shear wave velocity in the sample $c$ is evaluated from the time of flight $\tau$ using Eq. (14) and $c_{\mathrm{b}}=(1433 \pm 24) \mathrm{m} \cdot \mathrm{s}^{-1}$ $\left[c_{\mathrm{b}} \simeq\left(G_{\mathrm{b}}^{\prime} / \rho_{\mathrm{b}}\right)^{1 / 2}\right.$ since $G_{\mathrm{b}}^{\prime \prime} \ll G_{\mathrm{b}}^{\prime}$; see Appendix A].

Asay, J. R., Lamberson, D. L., and Guenther, A. H. (1969). "Pressure and temperature dependence of the acoustic velocities in polymethylmethacrylate," J. Appl. Phys. 40(4), 1768-1783.

Bagley, R. L., and Torvik, J. (1983). "Fractional calculus-A different approach to the analysis of viscoelastically damped structures," AIAA J. 21(5), 741-748.

Biwa, S., Ito, N., and Ohno, N. (2001). "Elastic properties of rubber particles in toughened pmma: Ultrasonic and micromechanical evaluation," Mech. Mater. 33(12), 717-728.

Cheeke, J. D. N. (2002). Fundamental and Applications of Ultrasonic waves (CRC Press, Boca Raton, FL).

Fokin, V., Ambati, M., Sun, C., and Zhang, X. (2007). "Method for retrieving effective properties of locally resonant acoustic metamaterials," Phys. Rev. B 76(14), 144302.

Hartmann, B., and Jarzynski, J. (1974). "Immersion apparatus for ultrasonic measurements in polymers," J. Acoust. Soc. Am. 56(5), 1469-1477.

Lefebvre, G., Wunenburger, R., and Valier-Brasier, T. (2018). "Ultrasonic rheology of visco-elastic materials using shear and longitudinal waves," Appl. Phys. Lett. 112, 241906.

Longin, P., Verdier, C., and Piau, M. (1998). "Dynamic shear rheology of high molecular weight polydimethylsiloxanes: Comparison of rheometry and ultrasound," J. Non-Newt. Fluid Mech. 76, 213-232. 
Papadakis, E. P. (1968). "Ultrasonic attenuation in thin specimens driven through buffer rods," J. Acoust. Soc. Am. 44(3), 724-734.

Pierre, J., Elias, F., and Leroy, V. (2013). "A technique for measuring velocity and attenuation of ultrasound in liquid foams," Ultrasonics 53(2), 622-629.

Reddy, S. S. S., Balasubramaniam, K., Krishnamurthy, C., and Shankar, M. (2005). "Ultrasonic goniometry immersion techniques for the measurement of elastic moduli," Compos. Struct. 67(1), 3-17.
Simonetti, F., and Cawley, P. (2004). "Ultrasonic interferometry for the measurement of shear velocity and attenuation in viscoelastic solids.," J. Acoust. Soc. Am. 115(1), 157-164.

Treiber, M., Kim, J.-Y., Jacobs, L. J., and Qu, J. (2009). "Correction for partial reflection in ultrasonic attenuation measurements using contact transducers," J. Acoust. Soc. Am. 125(5), 2946-2953.

Wu, J. (1996). "Determination of velocity and attenuation of shear waves using ultrasonic spectroscopy," J. Acoust. Soc. Am. 99(5), 2871-2875. 